Archivo Español de Arqueología, 2021, 94, e05

ISSN-L: 0066-6742 | eISSN: 1988-3110

https://doi.org/10.3989/aespa.094.021.05

NOTICIARIO

\title{
M. Agrippa ¿propietario de canteras de mármol en Carrara? Nueva nota lapicidinarum Lunensium hallada en el teatro romano de Gades
}

\author{
M. Agrippa: owner of marble quarries at Carrara? \\ A new nota lapicidinarum Lunensium from the Roman \\ theatre of Gades
}

\author{
Ángel Ventura Villanueva \\ Grupo de Investigación PAI-HUM 882 Antiguas ciudades de Andalucía, Universidad de Córdoba \\ aalvevia@uco.es \\ ORCID iD: https://orcid.org/0000-0003-0739-6231 \\ Juan de Dios Borrego de la Paz \\ Grupo de Investigación PAI-HUM 882 Antiguas ciudades de Andalucía, Universidad de Córdoba \\ juandepost@hotmail.com \\ ORCID iD: https://orcid.org/0000-0002-7655-3440 \\ Francisco Javier Alarcón Castellano \\ Coordinador RECA de Cádiz. Agencia Andaluza de Instituciones Culturales \\ francisco.alarcon.c@juntadeandalucia.es \\ ORCID iD: https://orcid.org/0000-0001-8122-1769
}

Enviado: 27-01-2021. Aceptado: 23-03-2021. Publicado online: 11-05-2021

Citar este artículo / Citation: Ventura Villanueva, Á., Borrego de la Paz, J. D. y Alarcón Castellano, F. J. (2021). "M. Agrippa ¿propietario de canteras de mármol en Carrara? Nueva nota lapicidinarum Lunensium hallada en el teatro romano de Gades". Archivo Español de Arqueología, 94, e05. DOI: https://doi.org/10.3989/aespa.094.021.05

RESUMEN: Damos a conocer una placa de mármol lunense bardiglio que conformó el pluteus de la prohedria en el teatro romano de Cádiz. En la cara inferior, con superficie tosca de extracción del bloque en cantera, porta la marca inscrita AGR seguida del numeral CXXXV que, de acuerdo con la hermenéutica de estas notae lapicidinarum, permite establecer el papel de Marco Agripa en la explotación de mármoles de Carrara. El empleo precoz de marmora importados en la construcción de este teatro hispano (27-13 a. C.) se explica por la relación de patronato sobre Gades de Agripa y Balbo el Menor.

Palabras clave: mármol lunense; arquitectura teatral hispana. 


\begin{abstract}
We present a bardiglio-marble plaque that formed the pluteus of the prohedria in the Roman theatre of Cádiz. On the underside, with a rough quarry extraction surface, it bears the inscribed mark AGR followed by the numeral CXXXV which, according to the hermeneutics of these notae lapicidinarum, allows confirming the role of Marcus Agrippa in the exploitation of Luni-Carrara marbles. The early use of imported marmora in the construction of this Hispanic theatre (27-13 BC) is explained by the patronage relationship over Gades of Agrippa and Cornelius Balbus Minor.
\end{abstract}

Keywords: marble quarries of Luna; architecture of Roman theatres in Spain.

Copyright: $\odot 2021$ CSIC. Este es un artículo de acceso abierto distribuido bajo los términos de la licencia de uso y distribución Creative Commons Reconocimiento 4.0 Internacional (CC BY 4.0).

Dedicado a Antonio Peña Jurado... siempre en la prohedria de nuestros corazones.

\section{EL DESCUBRIMIENTO Y SU CONTEXTO}

Durante las obras de adecuación del Centro de Interpretación del Teatro Romano de Cádiz, abordadas en 2009, se efectuaron tres sondeos arqueológicos emplazados estratégicamente para poder recabar información sobre los sectores de la orchestra y el proscaenium $^{1}$ (Fig. 1). Tales sondeos revelaron el arranque de una nueva scalaria radial sobre las dos primeras filas de gradas de la ima cavea (S-2), el límite de la prohedria (S-3) y el arranque del podio de la frons scaenae (S-4) (Alarcón, 2011).

Mas el Sondeo 3, de 1,4 m de diámetro, no solo localizó el basamento para el peldaño externo de la prohedria, sino también una gran placa de mármol caída a plomo tras el canal de inserción del pluteus. En su cara inferior, que no era visible cuando la barandilla marmórea estaba originariamente instalada en vertical, conservaba incisa la siguiente inscripción (Fig. 2):

\section{$\mathrm{L}$ (reversa) LATRO [[ $\overline{\mathrm{BA}}$ (reversas) $]] \overline{\mathrm{BAE}}$}

Desde un principio la interpretación estos epígrafes ha sido objeto de controversia (cfr. Ventura y Bo-

1 De la orchestra pudo averiguarse su cota absoluta al analizar un sondeo inédito (S-1) abierto en 2005 por R. Corzo, e. p. rrego, 2011 con Canto, 2012) ${ }^{2}$, si bien no cabe duda acerca del soporte material, realizado en mármol bardiglio de Luni-Carrara (Rodá et al., 2011), ni sobre la identificación del monograma BAE como signatio de los Baebii, familia relacionada con la explotación de dichas canteras marmóreas (especialmente las ubicadas en el distrito de Miseglia) y cuyas notae

2 Nosotros interpretamos el monograma BA, que sufrió damnatio memoriae mediante martellage, como abreviatura del destinatario o propietario por compra del mármol: BA(LBI); interpretación reforzada por los más recientes estudios respecto a la hermenéutica de estas notae Lunenses: Letta, 2015, p. 430. Y la palabra LATRO como insulto o apóstrofe dirigido al magnate gaditano, probable promotor del edificio: cfr. Strab. $3,5,3$ y Cic. Fam. 10,32. A. M. Canto, 2012 y previamente M. Mayer, 2011, p. 917, prefieren ver en Latro el cognomen de un artesano marmolista. Pero en contraposición debemos recalcar que, entre las numerosas notae lapicidinarum de las canteras de Carrara conocidas hasta la fecha, más de 200, ninguna muestra un cognomen desarrollado, utilizándose en todo caso siglas o abreviaturas: Paribeni y Segnenni, 2015a. Además del inusitado desarrollo del cognomen, el tamaño de las letras, el trazo ligero y la incisión más superficial de la palabra LATRO, suponen argumentos más que suficientes -a nuestro juicio- para reafirmar la existencia de dos inscripciones realizadas en momentos distintos por diferentes manos. Indicios estos que la interpretación ofrecida por A. M. Canto elude, presuponiendo sencillamente una confusión del lapicida al realizar el monograma BA, tachándolo y corrigiéndolo después $-\mathrm{O}$, tal vez, siendo corregido por una segunda mano-, en el definitivo BAE presente: Canto, 2012, pp. 57 y ss. Pensabene, 2015, p. 519, recoge ambas interpretaciones sin decantarse y fecha la pieza a comienzos de época augustea. 


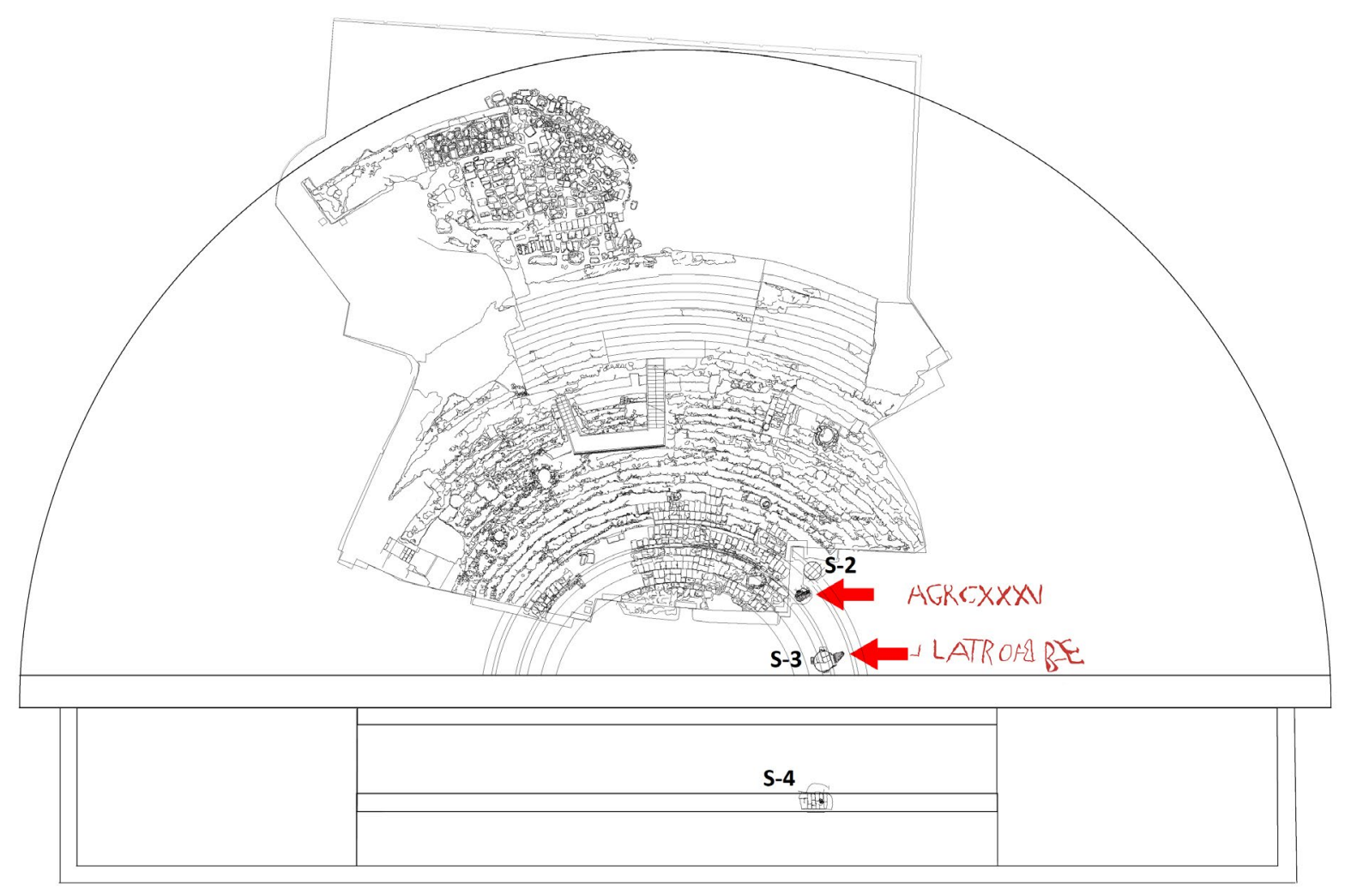

Figura 1. Planta del teatro de Gades. Lugares de hallazgo de las placas del balteus inscritas (dibujo J. D. Borrego).

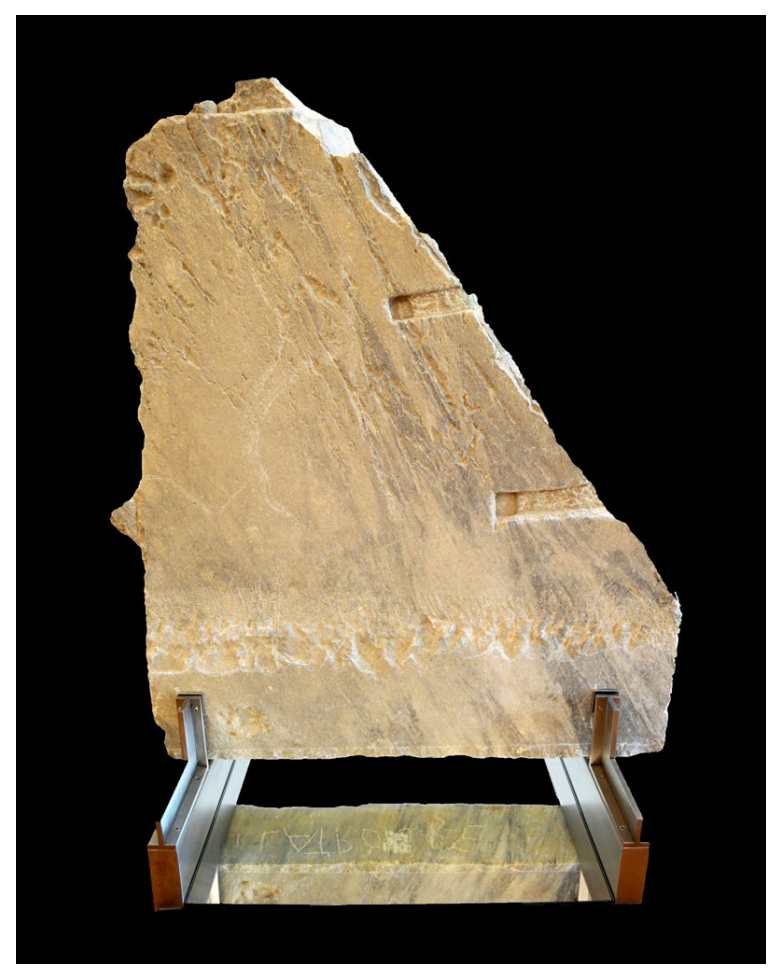

Figura 2. Placa de pluteus con nota BAE (fotografía J. D. Borrego). lapicidinarum son bien conocidas, tanto en bloques de extracción o semielaborados como en materiales puestos en obra (Paribeni y Segenni, 2003, 2015b, p. 400; Pensabene, 2004; Letta, 2015, p. 428; Gregori, 2016, p. 886; Paci, 2017, p. 506).

Ya en la publicación de esta placa de barandilla marmórea, que delimitaba la prohedria y servía de respaldo a los subsellia lígneos de las localidades más prestigiosas de la cavea (Fig. 3), se avanzó el hallazgo de otra laja distinta, que también parecía caída in situ, conservándose restos del pasamanos redondeado en el perfil del Sondeo 2 (Alarcón, 2011, p. 147; Ventura y Borrego, 2011, fig. 6). Dicha pieza, que no pudo extraerse entonces, volvió a localizarse en 2013, durante el seguimiento arqueológico derivado de la construcción de arcos de descarga bajo las medianeras de las viviendas colindantes con el área del teatro conservado a cielo abierto. En esta ocasión se descubrió parcialmente la base de dicha placa, donde podía verse con claridad un numeral inscrito (CXXXV), al que precedían dos letras de dudosa lectura (Alarcón, 2014). Finalizada esta intervención la pieza quedó in situ, embutida en el perfil y cubierta con geotextil para su protección. 


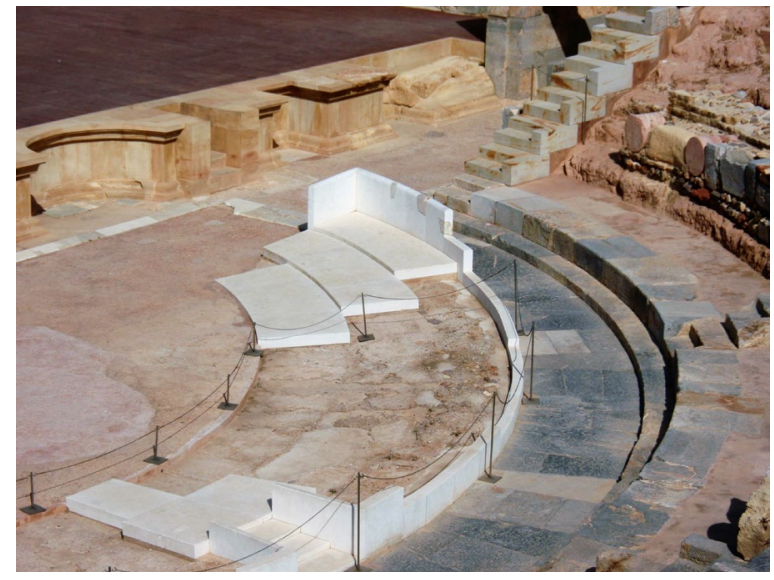

Figura 3. Colocación de las placas del pluteus de la prohedria en el teatro romano de Cartagena (fotografía A. Ventura).

Durante la campaña de 2017 se liberó todo el espacio existente entre la zona de la orchestra, excavada por la Universidad de Cádiz en 2011 (Borrego y Rodríguez, 2012; Bernal et al., 2014), y las primeras filas del graderío. Asimismo, se excavó el intradós de los arcos de descarga de los edificios colindantes, siendo durante el desarrollo de esta actividad, bajo el Arco 1, cuando volvió a aparecer la pieza de balteus ya vista en años anteriores. Decidida su extracción, se excavó el perfil hasta liberarla por completo, se numeraron los fragmentos que la componían y se almacenaron con el resto de materiales arqueológicos recuperados en dicha campaña (Borrego, 2020) (Fig. 4).

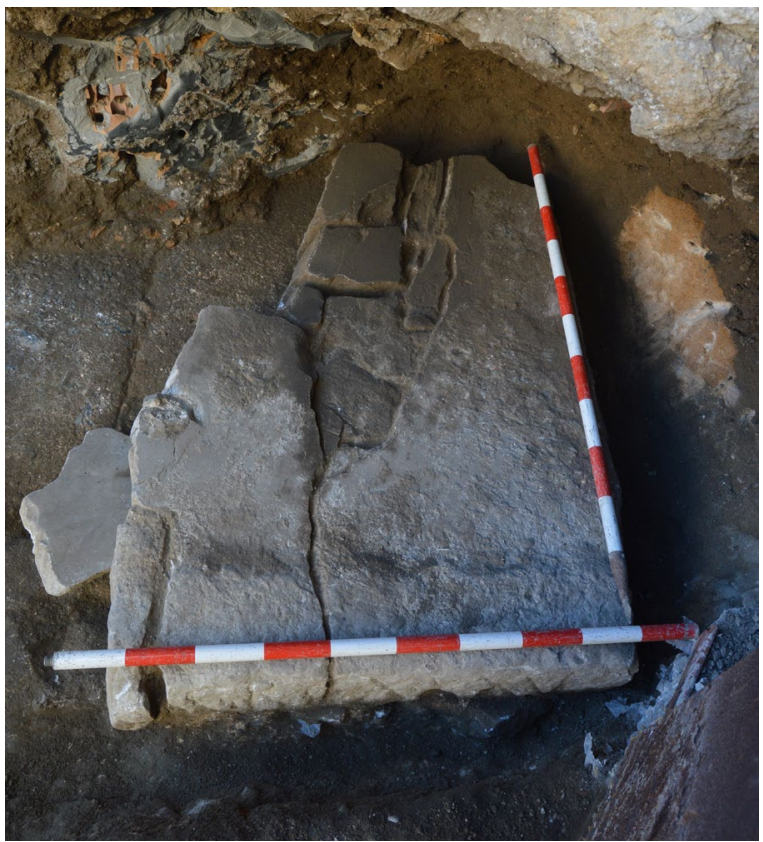

Figura 4. Momento de extracción de la placa con nota AGR (fotografía F. J. Alarcón).
En otoño de 2020, con vistas a los actos de conmemoración del $40^{\circ}$ aniversario del descubrimiento del monumento, se procedió a la limpieza y restauración de esta singular pieza en el Museo de Cádiz, pudiendo abordar entonces el estudio de la nueva marca de cantera $^{3}$ (Fig. 5, izquierda).

\section{DESCRIPCIÓN DEL SOPORTE Y DE LA NOTA}

Se trata de una placa rectangular de mármol blanco-azulado y grano muy fino, con vetas longitudinales más oscuras, que desde el punto de vista macroscópico resulta idéntico al de la placa con monograma BAE perteneciente a la misma construcción y, por eso, podemos identificar como la variedad bardiglio de las canteras de Luni-Carrara ${ }^{4}$. Aun cuando fracturada en múltiples fragmentos, de los que han desaparecido algunos de las esquinas superiores, conserva las dimensiones totales: $90 \mathrm{~cm}$ de altura, $82,5 \mathrm{~cm}$ de anchura y $14 / 15 \mathrm{~cm}$ de espesor (arriba y abajo, respectivamente).

La losa se obtuvo mediante el corte con sierra lapídea, comenzando por arriba, de manera que subsisten las rebabas de extracción final con cuñas en las partes inferiores de ambas caras principales vistas. De estas, la que miraba a la orchestra muestra abajo una franja toscamente desbastada y regularizada a gradina, de 29,5 $\mathrm{cm}$ de altura (1 pes), que sirvió para ajustar ahí el peldaño marmóreo de la fila externa de la prohedria. La parte superior muestra un acabado redondeado delimitado por sendos biseles, para funcionar como pasamanos. En la parte central de este se aprecian varias grietas, que siguen las vetas del mármol meteorizadas por el paso del tiempo, y que requirieron de una restauración antigua mediante grapa metálica de $29 \mathrm{~cm}$ de longitud, de la que subsisten solo la mortaja y los orificios cuadrados de anclaje (Fig. 5, derecha). Al igual que sucedía con la placa recuperada en 2009 , también reparada de antiguo con 3 grapas, este defecto favoreció que la pieza no fuera expoliada tras el abandono del teatro, como sí sucedió con el resto de losas del pluteus y del pavimento de la praecinctio y la orchestra. Las caras laterales muestran una anathyrosis perimetral, para ajuste con otras losas adyacentes de la misma barandilla. La

3 Noticia de prensa sobre la presentación de la pieza, con motivo de dicha efeméride: "El Teatro Romano de Balbo que también pudo ser de Agripa", Diario de Cádiz, 21 de noviembre de 2020: https://www.diariodecadiz.es/ocio/Teatro-Romano-Balbo-Agripa-arqueologia-cadiz_0_1521748085.html

4 Hemos enviado muestras al Laboratorio de Estudios Lapídeos del ICAC (Dra. Anna Gutiérrez) para la completa caracterización físico-química de esta variedad marmórea y concretar su procedencia dentro de los distritos marmoríferos lunenses. 

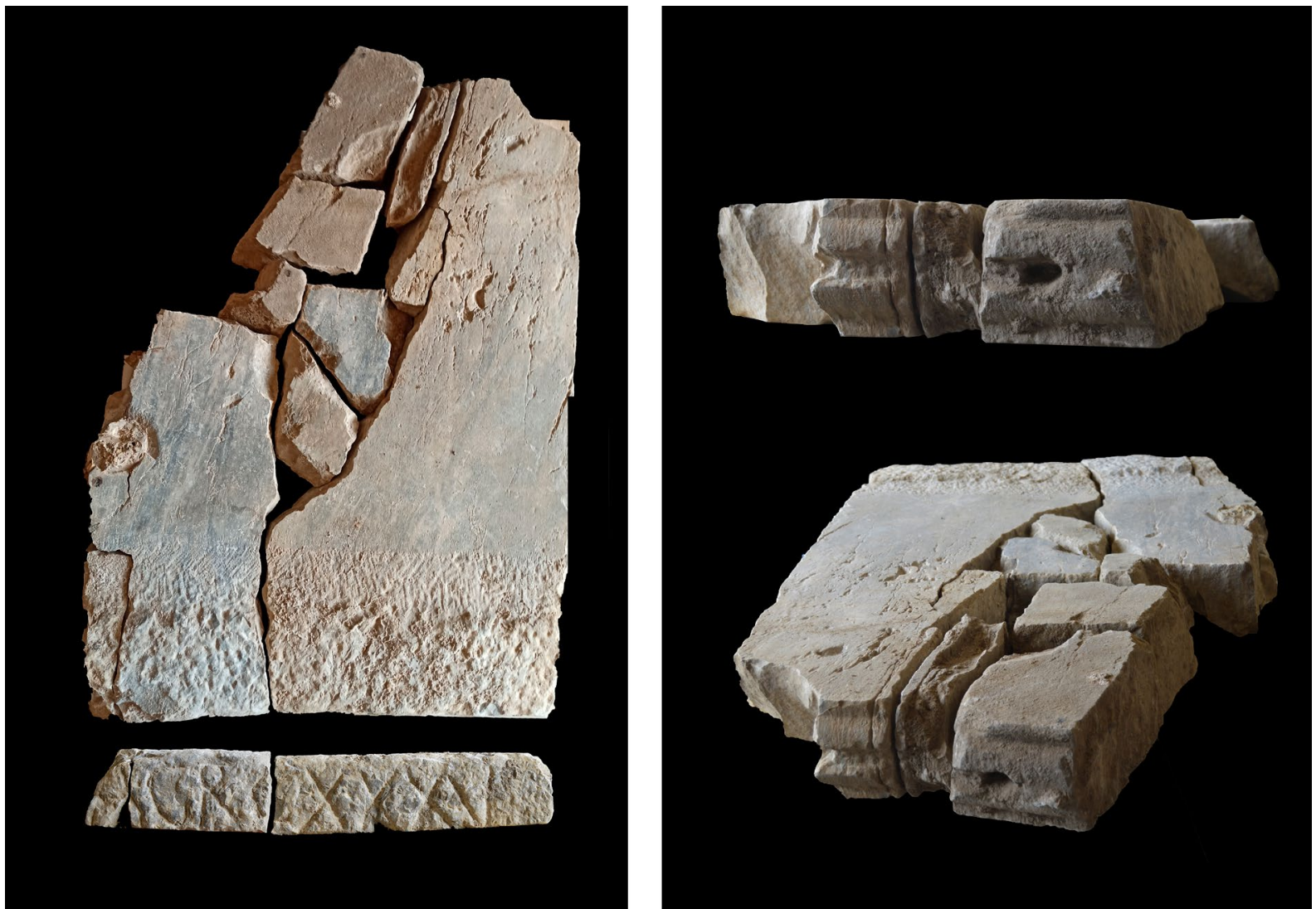

Figura 5. Placa con nota AGR: vista del frente, base inscrita y dorso con pasamanos y grapa de reparación (fotografías J. D. Borrego).

cara inferior, que no era vista una vez instalada la pieza, muestra una superficie tosca fruto de la caesura del bloque en cantera. Aquí, con letras de 11-12 cm de altura y ejecución grosera a puntero, se conserva la nota lapicidinarum con el siguiente texto (Fig. 6, abajo):

\section{AGR CXXXV (vacat)}

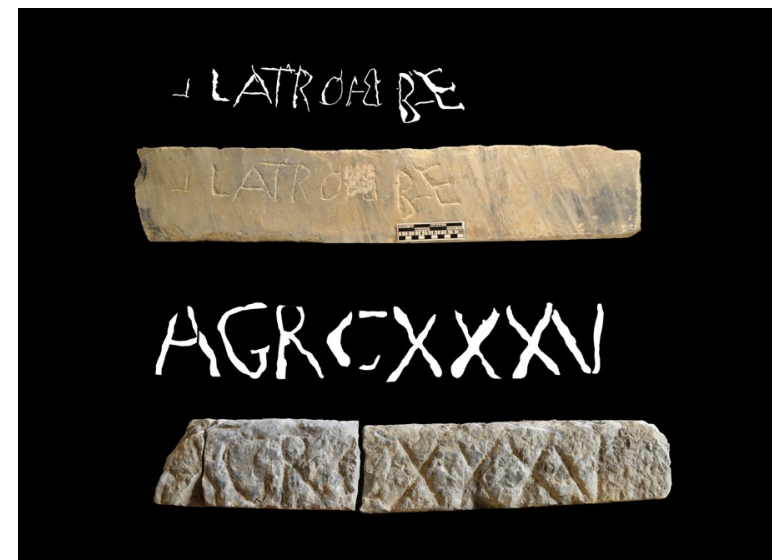

Figura 6. Las dos notae lapicidinarum recuperadas en el teatro de Gades (fotografia J. D. Borrego).
Parece razonable pensar que la inscripción se realizó en la propia cantera una vez cortada la placa con la serra, a juzgar por el correcto ajuste del texto a la escasa superficie disponible. Un paralelo muy cercano para esta nota lo encontramos en el teatro romano de Carthago Nova, inaugurado entre los años 5 y 1 a. C. En la cara inferior toscamente desbastada de una placa perteneciente también al pluteus, esta vez elaborado con la variedad blanca de Carrara, figura el texto: TV XXXIII (Soler, 2020, p. 170 y fig. 12).

5 En este caso la autora opina que la marca inscrita se realizó antes del corte con sierra de la placa. Propone la lectura T $\cdot$ VXXXIII, de la que resulta un numeral incoherente. Con la ligera variante que nosotros planteamos, se corrige la cifra y las dos primeras letras TV podrían abreviar el gentilicio del propietario o arrendatario de la cantera. Y a este respecto, resulta muy sugerente la existencia de varios Turtellii en la epigrafía lunense de época julioclaudia: CIL XI, 6956a y 6963. Uno de ellos, de nombre M. Turtellius C.f. Rufus, fue tribunus militum bis y douvir de la colonia en tres ocasiones, durante época augustea: CIL XI, 1341. 


\section{INTERPRETACIÓN}

Opinamos que las letras AGR de la nueva nota gaditana constituyen la abreviatura de AGR(IPPAE), por varias razones:

$\left.1^{\mathrm{a}}\right)$ De acuerdo con Cesare Letta (2015, pp. 428429), en esas 3 letras que anteceden al numeral, identificativo del bloque o placa concreto de una partida (probatio), se encierra el productor del mármol: propietario privado de una cantera, o arrendatario, en caso de situarse esta en terrenos públicos de la colonia de Luna.

$\left.2^{a}\right)$ Muy pocos nombres romanos comienzan con esa secuencia de letras: los gentilicios Agrius, Agrinius o Agrilius (muy raros) y los cognomina Agrestis, Agricola, Agrippinus, Agrippianus o Agrippa. Ninguno de tales nombres aparece abreviado, como en nuestro caso, en otras inscripciones del imperio ${ }^{6}$, excepto Agrippa: en un solo ejemplo que alude, sin dudas, a M. Vipsanius Agrippa ${ }^{7}$.

$3^{a}$ ) Ninguno de dichos nombres está documentado en las notae lapicidinarum de las canteras de Carrara (Paribeni y Segenni, 2015a, pp. 375-397). Y tampoco comparecen, a día de hoy, en la epigrafía lunense en general, salvo el caso de Agrippa $^{8}$.

$\left.4^{a}\right)$ M. Agrippa, mano derecha de Augusto y posteriormente su yerno, tenía intereses en la explotación de mármoles preciados. Poseía canteras de pavonazzetto en Docimium (Frigia) y de giallo antico en Chemtou (Numidia) (Pensabene, 1975, p. 186 y 2020, pp. 120 y 123). En este último distrito marmorífero existían, en época imperial, dos grandes officinae, denominadas respectivamente Regia y Agrippae, que probablemente obedezcan al inicio de la explotación a inicios del s. I a. C. bajo autoridad de los reyes númidas, la primera ${ }^{9}$, y a

6 Tenemos la abreviatura AGRIC(OLA) en CIL XI, 6689 y CIL III, 5647; AGRICOL(A) en CIL III, 11796, CIL VI, 30890, AE 1993, 587a y AE 2000, 533. En AE 1994, 1424 se abrevia un oficio como AGRI(MENSOR). Y solo en CIL I, 3612 encontramos la abreviatura del gentilicio AGRIL(IA).

7 EDCS n ${ }^{\circ}$ 74700603: tessera de hueso con fecha consular del año 27 a. C.: "IX K(alendas) Apr(iles) Imp(eratore) C(aesare) VII M(arco) Agr(ippa) III". En lingotes recuperados en el pecio de Comacchio y en Menorca su cognomen aparece abreviado AGRIP en estampillas impresas en frío, que testimonian la importación o, tal vez incluso, la producción de plomo hispano por su parte: Rodá, 2004.

8 En los c.d. fasti imperiali di Luna, AE 1988, 564 y AE 2008, 509, que citan a M. Agrippa investido con la tribunicia potestas $V$, correspondiente al año 14-13 a. C.; y en los fastos de un collegium fabrum que portan la fecha consular del año 22 d.C. mencionando al cónsul D. Haterius Agrippa: CIL XI, 1356.

9 Como testimonia la importación a Roma de esta variedad marmórea desde el año 78 a. C. según el testimonio de Plin. N.H. 36,49: "M. Lepidus Q. Catuli in consulatu conlega primus omnium limina ex Numidico marmore in domo posuit magna reprensione. is fuit consul anno urbis DCLXXVI. hoc primum invecti Numidici marmoris vestigium invenio, non in columnis tamen crustisve, ut supra Carystii, sed in massa ac vilissimo liminum usu". la apertura de nuevos frentes bajo la autoridad de Augusto y Agripa tras la anexión del reino a la provincia de Africa Nova y la instalación de la colonia Iulia Augusta Numidica Simitthensium en 27 a. C., la segunda. En las marcas de cantera de este distrito encontramos notae con la misma abreviatura, cuya resolución está fuera de toda duda, al aparecer otras veces desarrollada:

$$
\begin{aligned}
& \text { [Of(ficina)] Agr(ippae), en CIL VIII, } 14564 \text {, } \\
& \text { [Of(ficina) A]gripp(ae), en CIL VIII, } 14582 \text { y } \\
& \text { Of(ficina) Agrippae, en CIL VIII, } 14580 \text { y } 14581 .
\end{aligned}
$$

$\left.5^{\mathrm{a}}\right)$ El uso del cognomen, en lugar del gentilicio (como vemos en el caso de los Baebii o sugerimos con los Turtellii), para la signatio de los bloques o placas extraídos en las canteras propiedad de Agripa resulta comprensible en su caso específico, a tenor del testimonio de Séneca el Viejo ${ }^{10}$. De hecho, el nomen Vipsanius, que denotaba sus humildes orígenes familiares, no comparece en ninguna inscripción por él comandada o a él dedicada (salvo para denominar a sus hijas o libertos).

$6^{\text {a })}$ Que poseyera también canteras en Luni-Carrara era, podría decirse, la última pieza del rompecabezas, il tassello che mancava. Máxime cuando fue uno de los máximos responsables de la monumentalización y marmorización de la propia Roma -alentado por $\mathrm{Au}$ gusto-, como indican las fuentes literarias ${ }^{11}$ ya desde su edilidad desempeñada en 33 a. C. (Kienast, Eck y Heil, 2017, p. 65). Entonces, en el plazo de un solo año, pudo decorar las fuentes de Roma con 400 columnas marmóreas: invictum miraculum comprensible solamente si aceptamos un estricto control de los recursos lapídeos por su parte ${ }^{12}$.

$7^{\mathrm{a}}$ ) La investigación actual opina que se produjo la deductio de una colonia triunviral en Luna, probablemente entre 36 y 33 a. C. con veteranos de la

10 Sen. Contr. II, 6: "Tanta autem sub divo Augusto libertas fuit, ut praepotenti tunc M. Agrippae non defuerint qui ignobilitatem exprobrarent. Vipsanius Agrippa fuerat, (at) Vipsani nomen quasi argumentum paternae humilitatis sustulerat et M. Agrippa dicebatur. cum defenderet reum, fuit accusator qui diceret: 'Agrippae, Marce et quod in medio est' (voluit Vipsanium intellegi)...”.

11 Suet. Aug. 28,3: "Urbem neque pro maiestate imperio ornatam et inundationibus incendiisque obnoxiam excoluit adeo, ut iure sit gloriatus marmoream se relinquere, quam latericiam accepisset". Suet. Aug. 29, 4-5: "Sed et ceteros príncipes viros saepe hortatus est, ut pro facultate quiaque monimentis vel novis vel refectis et excultis urbem adornarent. Multaque a multis tunc extructa sunt, sicut... a Cornelio Balbo theatrum,... a M. vero Agrippa complura et egregia".

12 Plin. N.H. 36,121: "Sed dicantur vera aestimatione invicta miracula... Agrippa vero in aedilitate adiecta Virgine aqua ceterisque conrivatis atque emendatis lacus DCC fecit, praeterea salientes D, castella CXXX, complura et cultu magnifica, operibus iis signa $\mathrm{CCC}$ aerea aut marmorea inposuit, columnas e marmore $\mathrm{CCCC}$, eaque omnia annuo spatio". 
batalla de Naulocos (Sangriso, 1999) ${ }^{13}$. En ese momento pudieron haberse asignado a Agripa y al propio Augusto terrenos del ager Lunensis ubicados en los mejores distritos marmoríferos ${ }^{14}$. Otras parcelas habrían quedado en propiedad de la colonia, para la obtención de vectigalia con su locatio-conductio a particulares y, algunas menos, en fin, pudieron haberse repartido a veteranos de la nueva élite colonial, impulsándose así un sistema de explotación mixto, estatal-imperial, público-municipal y privado (Angeli, 2000; Pensabene, 2013, pp. 12-14 y 2015, pp. 453-457; Segenni 2015, pp. 441-450).

$8^{\text {a) }}$ M. Agrippa fue nombrado patronus y parens del municipio gaditano entre 27 y 18 a. C. (RPC I, 77-81; García Fernández, 1991, pp. 33-35) ${ }^{15}$. Además, es muy probable que visitara la ciudad durante su estancia en Hispania entre los años 19 y 18 a. C. Esta posibilidad se fundamenta en las propias acuñaciones locales antedichas, con marcado carácter conmemorativo de un evento singular. Una de ellas (RPC I, 77) decora su anverso con una imagen de él sentado en sella curulis y con la mano diestra extendida en gesto de adlocutio, a modo de "relieve histórico". En esos años realizó las tareas de medición de la Península Ibérica con las que componer su famoso orbis pictus y para las que Gades constituía un "punto geodésico" fundamental, como columna de Hércules en Europa y caput Oceani ${ }^{16}$. En

13 Liber Coloniarum (Grom. Vet. pp. 213, 6, L. y 223, 14, L.): "Colonia Florentina deducta a triumviris, adsignata lege Iulia... Ager Lunensis ea lege qua et ager Florentinus..."

14 Las canteras propiedad de Agrippa, tanto las de Luna (bardiglio) como las de Docimium (pavonazzetto) o Simitthus (giallo antico), tras su muerte, habrían pasado a formar parte del patrimonio de Augusto por herencia, como sugiere Pensabene, 1975, p.186 y apoyan los pasajes de Suetonio, Aug. 101,3: “...quamvis viginti proximis annis quaterdecies milies ex testamentis amicorum percepisset..." y Aug. 66,4: "Legata vel partes hereditatium a quibuscumque parentibus relicta sibi aut statim liberis eorum concedere aut, si pupillari aetate essent, die virilis togae vel nuptiarum cum incremento restituere consueverat".

15 En algunas de estas acuñaciones aparece como COS III, último consulado que desempeñara en 27 a. C.; pero en ninguna se alude a la tribunicia potestas, que le otorgó Augusto a partir del $1^{\circ}$ de julio del año 18 a. C. y supuso, de facto, su asociación al poder imperial: Kienast, Eck y Heil, 2017, pp. 65-66.

16 Plin. N.H. 3,16-17: "Longitudinem universam eius prodidit M. Agrippa CCCCLXXV p., latitudinem CCLVIII... Baeticae longitudo nunc a Castulonis oppidi fine Gadix CCL et a Murgi maritima ora XXV p. amplior, latitudo a Carteia Anam ora CCXXXIIII p. Agrippam quidem in tanta viri diligentia praeterque in hoc opere cura, cum orbem terrarum orbi spectandum propositurus esset, errasse quis credat et cum eo Divum Augustum? is namque conplexam eum porticum ex destinatione et commentariis M. Agrippae a sorore eius inchoatam peregit". Sobre las tareas cartográficas de Agripa: Nicolet, 1989, pp. 95-121. el marco de tal relación de patronato resulta lógico que donara mármol -al menos 135 placas-, a su ciudad cliente como acto evergético (beneficium); o bien se lo facilitara a su amigo Balbo el Menor, promotor por entonces de la construcción de la neapolis gaditana ${ }^{17}$ (Rodríguez Neila, 1992, pp. 289-297).

En la edificación del grandioso teatro de $\mathrm{Ga}$ des, con un diámetro de $118 \mathrm{~m}$ y ubicado en la isla de Kotinoussa, asiento de dicha neápolis (Borrego 2013), se emplearon preciados mármoles de importación en un momento muy precoz para la arquitectura hispano-romana: años 20 a. C., atendiendo al estilo "segundo triunvirato" con que se labraron los capiteles de la scaena y otros elementos de su decoración arquitectónica (Borrego, 2011). Así, en la fase fundacional del edificio, la orchestra aparecía pavimentada y delimitada con bardiglio, el pulpitum y el podio de la frons scaenae revestidos de mármol blanco lunense, con el que también se elaboraron las basas, capiteles y entablamentos de la columnatio de la scaena, dotada de fustes de africano (marmor Luculleum) y alabastro fiorito (onyx) (Bernal et al., 2014; cfr. Loza y Beltrán, 2018). Este último detalle refuerza la atribución del monumento a Balbo el Menor, quien también decoró con 4 columnas de ónice la escena del teatro levantado por él en el Campo de Marte ${ }^{18}$.

El año 19 a. C. se nos desvela ahora como especialmente significativo para el municipio de $G a$ des y su edificio de espectáculos. Si Agripa hubiera sido nombrado patrono a mediados de los años 20, poco después de desempeñar el tercer consulado, proporcionando entonces los mármoles para el teatro, este bien pudo finalizarse (dedicatio) para ser usado con motivo de su visita a la ciudad el 19 a. C.; año en que Balbo, el otro comitente, celebraba el triunfo en Roma ${ }^{19}$ (Rodríguez Neila, 1992, p. 280). Si el nombramiento como patrono y

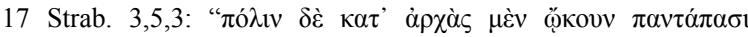

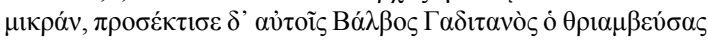

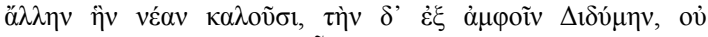

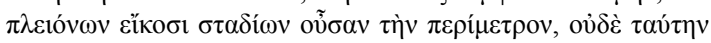

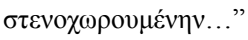

18 Plin. N.H. 36,60: "variatum in hoc lapide (i.e. onyx) et postea est, namque pro miraculo insigni quattuor modicas in theatro suo Cornelius Balbus posuit; nos ampliores XXX vidimus in cenatione, quam Callistus Caesaris Claudi libertorum, potentia notus, sibi exaedificaverat. hunc aliqui lapidem alabastriten vocant, quem cavant et ad vasa unguentaria, quoniam optume servare incorrupta dicatur". El theatrum Balbi de Roma se inauguró el 13 a. C.: Dio 54,25,2.

19 Concretamente el 27 de marzo, como testimonian los Fasti triumphales Capitolini: "L. Cornelius P.f. Balbus procos. a. DCCXXXIV ex Africa VI k. april.” 
la consecuente donación marmórea tuvo lugar más tarde, coincidiendo con la visita, las obras habrían comenzado por entonces (inauguratio) y el teatro gaditano sería contemporáneo al de Emerita, patrocinado íntegramente por Agripa -aunque sin uso de mármol en la fase fundacional-y concluido en 1615 a. C. (Stylow y Ventura 2018, pp. 157-163). En cualquier caso, la nueva marca de cantera asegura que la construcción del teatro gaditano se abordó con anterioridad a marzo del año 12 a. C., fecha de su muerte. Se trata del edificio más antiguo de Hispania en el que se constata un empleo masivo de mármol lunense (Pensabene, 2004; Gutiérrez y Rodá, 2012; Pensabene, 2013) y otros marmora de importación (Fig. 7), anterior a los teatros de Tarraco, Carthago Nova, Italica o Corduba.

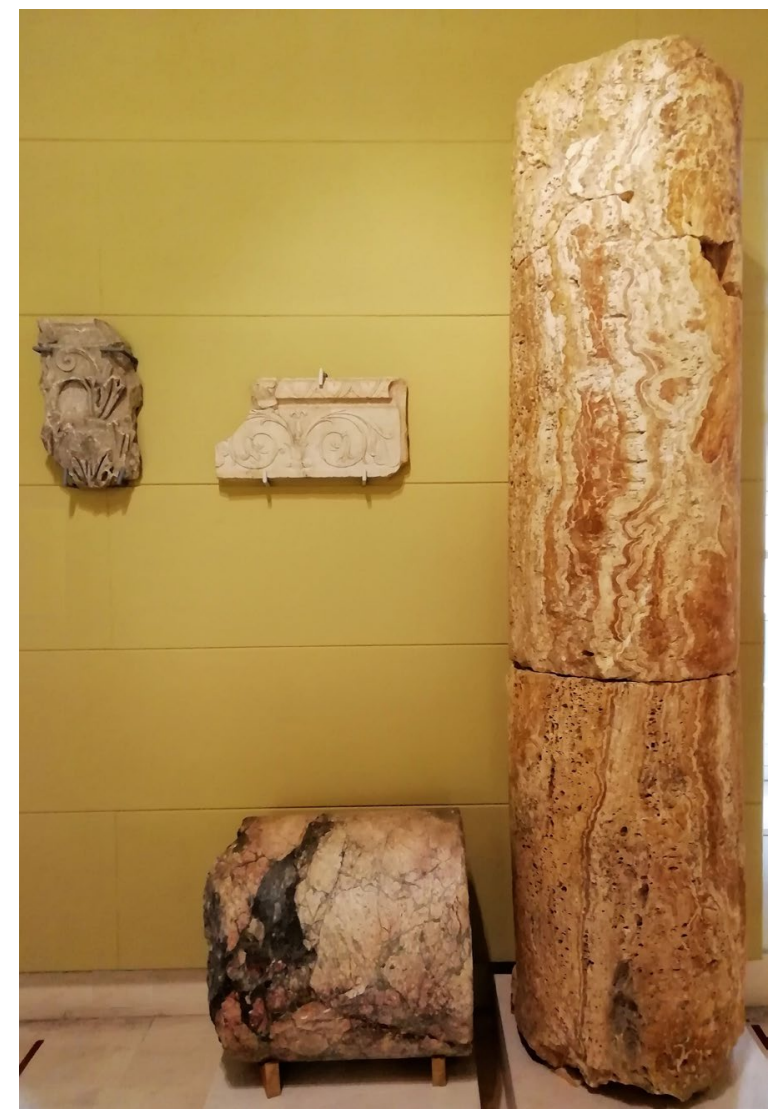

Figura 7. Decoración arquitectónica marmórea del teatro de Gades capitel y relieve del pulpitum labrados en blanco de Carrara; fustes labrados en alabastro y africano (fotografía A. Ventura).

\section{FUENTES INÉDITAS}

Alarcón, F. (2014). Memoria de la actividad arqueológica preventiva en el teatro romano de Cádiz. Control arqueológico de apoyo a la obra de emergencia (segunda fase). Informe inédito depositado en la Delegación territorial de la Consejería de Cultura y Patrimonio Histórico en Cádiz.

Borrego, J. D. (2020). Memoria final de los trabajos arqueológicos en el teatro romano de Cádiz y Posada del Mesón (Cádiz 2017). Informe inédito depositado en la Delegación territorial de la Consejería de Cultura y $\mathrm{Pa}$ trimonio Histórico en Cádiz.

Borrego, J. D. y Rodríguez, O. (2012). “Análisis arquitectónico de los restos del teatro romano" en Bernal D. y Arévalo, A. (Dir.) Informe y memoria definitiva de la actividad arqueológica preventiva en el teatro romano de Cádiz excavaciones arqueológicas de apoyo a las obras de emergencia (2010 - 2011). Informe inédito depositado en la Delegación territorial de la Consejería de Cultura y Patrimonio Histórico en Cádiz. pp. 165-223.

\section{BIBLIOGRAFÍA}

Alarcón, F. (2011). "Excavación de Pozos de Observación en el Centro de Interpretación del teatro Romano de Cádiz". En: Bernal, D. y Arévalo, A., eds., El Theatrum Balbi de Gades. Cádiz: Universidad de Cádiz, pp. 141-153.

Angeli Bertinelli, M. G. (2000). "Lunensis epigraphica: un magistrato della colonia di Luna fra impegno pubblico e imprenditoria privata”. En: Paci, G. (Ed.). Epigraphai. Miscellanea epigrafica in onore di Lidio Gasperini. Tivoli: Editrice Tipigraf, pp. 29-41.

Bernal, D., Arévalo, A., Bustamante, M., Sánchez, V., Lara, M., Vargas, J. M., Borrego, J. D., Rodríguez, O., Ventura, A. y Alarcón, F. J. (2014). "Del Theatrum Balbi de Gades. Recientes excavaciones arqueológicas (2010-2012)”. En: Álvarez, J. M., Nogales, T. y Rodà, I. (Eds.). Actas del XVIII Congreso Internacional de Arqueología Clásica. Centro y periferia en el mundo clásico, vol. I. Mérida: Museo Nacional de Arte Romano, pp. $847-851$

Borrego, J. D. (2011). "La configuración arquitectónica del teatro romano de Cádiz. Nuevas perspectivas”. En: Bernal, D. y Arévalo, A. (Eds.). El Theatrum Balbi de Gades. Cádiz: Universidad de Cádiz, pp. 171-226.

Borrego, J. D. (2013). La génesis del modelo arquitectónico teatral en la Bética. El teatro romano de Cádiz. Tesis Doctoral, Universidad de Córdoba.

Canto, A. M. (2012). “Cádiz". En: Hispania Epigraphica 18, 2009, no 92. Madrid: Universidad Complutense, pp. 55-61.

Corzo, R. (en prensa). El teatro romano de Gades. Veinticinco años de excavaciones arqueológicas (1980-2005). Sevilla.

García Fernández, E. (1991). "El ius Latii y los municipia latina". Studia historica. Historia antigua, 9, pp. 29-41.

Gregori, G. L. (2016). "Recensione a Paribeni, E. y Segenni, S., Notae lapicidinarum dalle cave di Carrara, Pisa 2015". Archeologia Classica LXVII - n.s. II, 6, pp. 883-887. 
Gutiérrez, A. y Rodá, I. (2012). "El mármol de Luni-Carrara en la fachada mediterránea de Hispania". En: Keay, S. (Ed.). Rome, Portus and the Mediterranean. Archaeological Monographs of the British School at Rome 21. London, pp. 293-312.

Kienast, D., Eck, W. y Heil, M. (2017). Römische Kaisertabelle. Grundzüge einer römischen Kaiserchronologie. 6., überarb. Aufl. Darmstadt.

Letta, C. (2015). "Tipologia delle notae apposte nell'area delle cave lunensi”. En: Paribeni, E. y Segenni, S. (Eds.). Notae lapicidinarum dalle cave di Carrara. Pisa: Pisa University Press, pp. 425-432.

Loza, M. L. y Beltrán, J. (2018). "El uso de los travertinos calcíticos en las provinciae Tarraconensis y Baetica. Una aproximación general" en Beltrán, J, Loza, M. L. y Ontiveros, E. (Coords.). Marmora Baeticae. Usos de materiales pétreos en la Bética romana. Estudios arqueologicos y análisis arqueométricos. Spal, Monografías Arqueología XXVII. Sevilla, pp. 137-149.

Mayer, M. (2011). "La presencia y explotación de marmora en la Hispania romana: algunas notas epigráficas". En: Brandt, O. y Pergola, P. (Eds.). Marmoribus vestita. Miscellanea in onore di Federico Guidobaldi, vol. II. Città del Vaticano: Arbor Sapientiae, pp. 911-922.

Nicolet, C. (1989). L'inventario del mondo. Geografia e politica alle origini dell'impero romano. Roma-Bari: Laterza.

Paci, G. (2017). "Recensione a Paribeni, E. y Segenni, S., Notae lapicidinarum dalle cave di Carrara, Pisa 2015". Epigraphica LXXIX, pp. 502-507.

Paribeni, E. y Segenni, S. (2003). "Iscrizioni su manufatti semilavorati delle cave lunensi". En: Angeli Bertelli, M.G. y Donati, A., eds., Usi e abusi epigrafici. Atti del colloquio internazionale di epigrafia latina (Genova 2001). Serta antiqua et medievalia, vol. VI. Roma: Arbor Sapientiae, pp. 65-79.

Paribeni, E. y Segenni, S. (2015a). Notae lapicidinarum dalle cave di Carrara. Pisa: Pisa University Press.

Paribeni, E. y Segenni, S. (2015b). "Commenti alle notae di Carrara”. En: Paribeni, E. y Segenni, S., (Eds.). Notae lapicidinarum dalle cave di Carrara. Pisa: Pisa University Press, pp. 399-413.

Pensabene, P. (1975). "Sull'impiego del marmo di Cap de Garde”. Studi Miscellanei, 22, pp. 179-190.

Pensabene, P. (2004). "La diffusione del marmo lunense nelle province occidentali”. En: Ramallo, S. F. (Ed.). La decoración arquitectónica en las ciudades romanas de
Occidente (Actas del Congreso Internacional, Cartagena, 8-10 octubre 2003), Murcia, pp. 421-443.

Pensabene, P. (2013). "Il marmo lunense nei programmi architettonici e statuari dell'Occidente romano". En: García-Entero, V. (Ed.). El marmor en Hispania: explotación, uso y difusión en época romana. Madrid: UNED, pp.17-48

Pensabene, P. (2015). "I marmi bianchi di Luni (Carrara)". En: Paribeni, E. y Segenni, S. (Eds.). Notae lapicidinarum dalle cave di Carrara. Pisa: Pisa University Press, pp. 451-520.

Pensabene, P. (2020). "Marmi e colonne nella Roma tardorepubblicana". En: Vinci, M.S., Ottati, A. y Gorostidi, D. (Eds.). La cava e il monumento. Materiali, officine, sistema di costruzione e produzione nei cantieri edilizi di età imperiale. Roma: Quasar, pp. 115-134.

Rodá, I. (2004): "Agripa y el comercio del plomo". Mastia 3, pp. 183-193.

Rodá, I., Álvarez, A., Gutiérrez, A. y Domènech, A. (2011). "Informe del análisis de un conjunto de muestras procedentes del Teatro Romano de Cádiz". En: Bernal, D. y Arévalo, A. (Eds.). El Theatrum Balbi de Gades. Cádiz: Universidad de Cádiz, pp. 222-226 y 254-256.

Rodríguez Neila, J. F. (1992). Confidentes de César. Los Balbos de Cádiz. Madrid: Sílex.

Sangriso, P. (1999): "La data delle colonie triumvirali di Luni e di Pisa. Note su CIL XI, 1330". Epigraphica LXI, pp. 47-50.

Segenni, S. (2015). "Propietà, amministrazione e organizzazione del lavoro nelle cave lunensi in età romana". En: Paribeni, E. y Segenni, S. (Eds.). Notae lapicidinarum dalle cave di Carrara. Pisa: Pisa University Press, pp. 441-450.

Soler, B. (2020). "El Teatro de Cartagena, Planificación, Proceso Constructivo y Valoración Económica de la Obra”. En: Courault, Ch. y Márquez, C. (Eds.). Quantitative Studies and Production Cost of Roman Public Construction. Córdoba: UCOpress, pp. 143-208.

Stylow, A. U. y Ventura, A. (2018). "Inscripciones asociadas a la scaena del Teatro". En: Mateos, P. (Ed.). La Scaenae Frons del Teatro Romano de Mérida, Anejos de Archivo Español de Arqueología LXXXVI. Madrid: IAM-CSIC, pp. 155-192.

Ventura, A. y Borrego, J. D. (2011). "Notae lapicidinarum lunensium, damnatio memoriae y graffito maldiciente en una inscripción del Teatro Romano de Gades". En: Bernal, D. y Arévalo, A. (Eds.). El Theatrum Balbi de Gades. Cádiz: Universidad de Cádiz, pp. 227-256. 